\title{
New approaches to the treatment of endometrial pathology in patients with extragenital diseases
}

\author{
DOI 10.52739/bio-up.2.2021.22-27
}

Ihor I. Chermak', Volodymyr I. Chermak², Roman M. Zakordonets ${ }^{2}$, Olori Sharon Oboghene ${ }^{3,}$ Anatolii Ya. Senchuk ${ }^{2}$

\begin{abstract}
With purpose to increase efficiency and decrease frequencies of complications after treatment of endo- and myometrium pathology in patients of the perimenopausal period with extragenital pathology this study has been conducted. Different conducted clinic-laboratory, instrumental and pathology examination were performed. 130 patients of the perimenopausal period from somatic pathology (46-60 years) were divided into representative groups. The main group consisted of 100 patients who suffered from endometrium hyperplasia and adenomyosis. The control group included 30 healthy women without pathology of endoand myometrium.
\end{abstract}

Patients of the main group, depending on the applied treatment-and-prophylactic approach, were divided into two subgroups. Group I included 50 women who underwent hysteroscopy and intrauterine cryosurgery, magnesium supplements, and thromboembolic complications (TEC) prevention measures. Patients of the II group (50 patients) underwent hysteroscopy and treatment and prevention measures following the recommendations of the Protocol of the Ministry of Health of Ukraine (№ 676 of 31.12.2004).

The effectiveness of the proposed approach to diagnosis, minimally invasive treatment (hysteroscopy and intrauterine cryosurgery), and prevention of TEC and inflammatory complications of endo pathology- and myometrium in patients of the perimenopausal period with extragenital pathology, according to the clinic-laboratory and cytomorphological examination, the results of

\footnotetext{
${ }^{1}$ Department of Obstetrics and Gynecology and Neonatology of the Institute of Postgraduate Education, Bogomolets National Medical University, Kyiv, Ukraine

2 Department of Obstetrics and Gynecology, Private Higher Education Establishment Kyiv Medical University, Kyiv, Ukraine

3 Department of Pathological Anatomy, Histology and Forensic Medicine, Private Higher Education Establishment Kyiv Medical University, Kyiv, Ukraine
} 
ultrasound was $82.0 \%$, prevention of TEC and inflammatory complications - $100 \%$ cases. According to generally accepted approaches, the effectiveness of treatment was $36.0 \%$, TEC prevention - $96.0 \%$, prevention of inflammatory complications - $78.0 \%$.

Keywords: endometrial hyperplasia, extragenital pathology, treatment, prevention of complications

\section{INTRODUCTION}

Statistical reports and data from the scientific literature indicate a steady increase in the high frequency of hyperplastic processes (HEP) and endometrial cancer (Sasaki, Andrade, \& Pereira, 2018), (Vitale, Haimovich, \& Carugno, 2021), (Sadigov, 2019), (Engstrom, \& Meyskens, 2005), (Vovk, \& Ponomareva, 2016), (Westin, \& Yates, 2021), Rotenberg, \& Goldberg, 2020). The most vulnerable to endometrial cancer are women of the perimenopausal period. More than half of the cases of this cancer result from the malignancy of HEP (Korniyenko, 2017), (Fiscella, \& Guzick, 2006), (Uglietti, Buggio, Farella, Chiaffarino, Dridi, Vercellini, \& Parazzini, 2019), (Wise, Gill, Lensen, Thompson, \& Farquhar, 2016), (Shalowitz, Goodwin, \& Schoenbachler, 2019).

The perimenopausal period is associated with the biological transformation of a woman's body, the reduction and subsequent exclusion of ovarian function. Against this background, there are a restructuring of the central autonomic nervous system, which has functioned in a cyclical mode in recent decades, manifest autonomic and psychoemotional disorders, deteriorating general health, increasing risk of intrauterine pathology (Korniyenko, 2015), (Pham, \& Mueller, 2019), neurovegetative, psychoemotional and metabolic endocrine disorders (Hervé, \& Céline, 2018), (Benyuk, \& Usevych, 2020), (Horak, \& Chmel,2012), (Clarke, $\&$ Wentzensen, 2020).

The urgency of the problem is also due to the fact that from $65 \%$ to $80 \%$ of perimenopausal women have various extragenital pathology, which is a contraindication to the use of specific hormone therapy and surgery (Gladchuk, Rozhkovskaya, \& Kosey, (2016), (Giannella, \& Paganelli, 2017).
The use of hormonal drugs for the treatment of precancerous diseases of the endometrium and myometrium has several contraindications, hormone therapy does not make sense for severe forms of precancerous pathology of the endometrium, and often its use causes severe complications, especially thromboembolic (Harvey \& Zimmerman, 2018), (Tatarchuk, Kosey, Zanko, Retunska, 2018), (Maggi, \& Limonta, 2016), (Tatarchuk, Kosey, Zanko, Retunska, 2018).

Radical methods (surgical interventions in the amount of supravaginal amputation and extirpation of the uterus) traumatic, usually lead to organ removal, surgical castration (frequency of ovariectomy after 45 years old $90.0 \%$, the following psychosomatic problems, associated with the loss of menstrual and reproductive function of women. In addition, surgical and mental trauma, harmful effects of anesthesia, restriction of motor activity in the postoperative period, and all this against the background of the presence of genital and somatic pathology in women, mostly after 45 years of life, are risk factors, which cause a high risk of thromboembolic complications (TEC) in this category of patients (Golyanovskyy, \& Budchenko, 2018), (Tatarchuk, Kosey, Zanko, Retunska, 2018), (Beelen, \& Bongers, 2021).

The above causes an unsatisfactory state of the question of timely, effective, recurrence-free, and safe treatment of connective pathology of the uterus and endometrium in women of the late reproductive period. Late request for help, repeated scraping without the subsequent appointment of adequate treatment, or no treatment at all lead to the recurrence of the disease, its progression to severe hyperplasia (adenomatosis), and endometrial cancer (Di Spiezio Sardo\& Bifulco, 2016), (Senchuk, Zakordonets, 2017).

The aim of the study: increasing the effectiveness and reducing the frequency of complications after treatment of and endo- and myometrium pathology in patients of the perimenopausal period with extragenital pathology due to optimization of approaches and organ-sparing therapy based on the results of a comprehensive examination of this pathology. 


\section{MATERIALS AND METHODS}

Clinical-laboratory, instrumental, and hardware examination of 130 patients of the perimenopausal period with somatic pathology (46-60 years), who were divided into representative groups. The main group consisted of 100 patients with HEP and adenomyosis. The control group included 30 healthy women without endometrial and myometrial pathology. Patients of the main group, depending on the applied treatment-and-prophylactic approach, were divided into two groups. Group I included 50 women who underwent hysteroscopy and intrauterine cryosurgery, magnesium supplements, and TEC prevention measures. Patients of the II group (50 patients) underwent hysteroscopy and treatment and prevention measures following the recommendations of the Protocol of the Ministry of Health of Ukraine (№ 676 of 31.12.2004).

Hysteroscopic interventions were performed according to the generally accepted method using a hysteron resectoscope "KARL STORZ" 26050IS, Germany, optics "HOPKINS."

Cryosurgical interventions were performed using the installation "Cryo-pulse," in which liquid nitrogen is used as a refrigerant. For the prevention of inflammatory complications in the postoperative period, prescribed broad-spectrum antibacterial drugs and rectal thrombolytic drugs are used. If patients have a medium or high risk of developing TEC, they were additionally prescribed a specific - low molecular weight heparins $(\mathrm{LMWH})$ - and nonspecific (elastic compression) prevention, as well as vascular drugs during 2-3 months.

The obtained results were achieved by statistical processing of materials using the analysis package of IBM SPSS Statistics v. 22.

\section{RESULTS AND DISCUSSION}

The age of the patients examined and treated by us varied from 46 to 60 years and averaged $54.2 \pm 6.7$ years in Basic and $53.1 \pm 5.9$ in the control group $(p>0.05)$.

Depending on the results of ultrasound and pathomorphological examination, all patients were distributed as follows: simple atypical
HE - 42 (42.0\%), complex atypical endometrium hyperplasia $(23.0 \%)$, polyps (glandular and glandular-fibrotic) endometrium - $35.0 \%$. All cases of endometrium hyperplasia were combined with adenomyosis of the 1st and 2nd degrees.

The structure of extragenital pathology prevailed: varicose veins of the lower extremities - 61 (61.0\%), arterial hypertension 2-3 stages - 46 (46.0\%); coronary heart disease, angina pectoris - $8(8.0 \%)$; obesity - 43 $(43.0 \%)$, liver disease, gallbladder and pancreas - $52(52.0 \%)$. In $78.0 \%$ patients with somatic pathology was represented by two or more diagnoses.

Peculiarities of the reproductive history of patients with hyperplasia of endometrium (HEP) in combination with adenomyosis are early menarche (up to 14 years in $85.3 \%$ of patients), a large number of pregnancies (four or more in $80.0 \%$ ), most of which ended in abortions (three or more in $68.0 \%$ ).

The study of the state of the hemostasis system in patients of the perimenopausal period with HEP and adenomyosis and extragenital pathology showed a deviation in the vascular-platelet system, which is manifested in a significant decrease in platelet count to 159.5 $\pm 12.7 \times 109 / /$ and an increase in aggregation capability $42,3 \pm 4.4 \%$ compared with the corresponding indicators in healthy women (respectively $226.5 \pm 11.3 \times 109 / /$ and $29.5 \pm$ $2.8 \%)$.

In the fibrinolysis system, a significant increase in plasma lysis $(p<0.05)$ was determined - from $154.3 \pm 9.1$ seconds in control to $213.9 \pm 11.2$ seconds in patients, a decrease in blood pressure-III (from $71.3 \pm 2,2 \%$ to 57.3 $\pm 3.3 \%$ ) and, compared with healthy women, a 3 -fold increase in soluble fibrin $(+)-3.9 \pm$ $0.4(p<0.05)$.

Such indicators indicate the maximum stress of the hemostasis system in patients with HEP and adenomyosis. Thus, the unstable equilibrium of the hemostasis system is inherent in the first phase (hypercoagulation) of the DIC syndrome. This is also confirmed by the fact that we observed a positive reaction in the ethanol test in $97.3 \%$ of patients with connective pathology of the endometrium and uterus, which 
differs significantly from that in healthy women $-30.7 \%$.

The results of a comprehensive examination of patients in the perimenopausal period with combined endo- and myometrium pathology on the background of extragenital pathology showed that thrombophilic significantly risk factors in this category of patients should include endo pathology- and myometrium, presumed surgery (hysteroscopy, intrauterine cryosurgery), age over 45 years, taking hormonal drugs, heart disease-vascular system (hypertension, varicose veins) and other severe extragenital pathology. Thrombogenic potential in patients with combined endo pathology- and myometrium increases in the presence of hemodynamic disorders in the affected organ, magnesium deficiency in the patient's body, and the presence of psychogenic disorders. The presence of these factors in combination with changes in the hemostasis system (vascular-platelet link, plasma hemostasis, and fibrinolysis system) indicated the presence of medium and high risk of TEC. It required thromboprophylaxis, which consisted of an additional purpose-specific - low molecular weight heparins (LMWH) and nonspecific (elastic compression, taking magnesium supplements) prevention and vascular drugs during 2-3- months.

The analysis of clinical results is carried out, laboratory, instrumental, immunohistochemical, psychological research, determination of the state of the hemostasis system before and through 1,3 and 6 months after hysteroscopy and endocryosurgical treatment of endometrial pathology in combination with adenomyosis showed that the method of treatment is applied, prevention of thromboembolic and inflammatory complications in these patients is highly effective.

The results of the study of the state of the hemostasis system one month after treatment showed a positive effect of the proposed method of treatment on this system. So, in the first place, in contrast to patients with HEP and adenomyosis (159.5 $\pm 12.7 \times 109)$, normalized platelet count $(189.7 \pm 9.1 \times 109)$ did not differ from the platelet count in healthy women $(226.5 \pm 11.3 \times 109)$. The indicator of their aggregation evidenced the normalization of platelet aggregation. Indicators after treatment $(29.7 \pm 4.1 \%)$ significantly decreased $(p<0.05)$ and approached the state of platelet aggregation in healthy people $(29.5 \pm 2.8 \%)$ women $(p>0.05)$.

One month after applying our proposed treatment (1st group) indicated its benefits, significant positive shifts in the fibrinolysis system. However, indicators of plasma lysis $(161.8 \pm 5.8)$ and blood pressure-llI $(65.9 \pm 2.1)$ were almost no different from healthy women (in accordance $154.3 \pm 9.1$ and $69.3 \pm 2.2$ ). On the contrary, after conventional therapy (2nd group), fibrinolysis rates were significant $(\mathrm{p}<0.05)$ the worst (plasma lysis (sec) $227.9 \pm 7.2$; BP-III (\%) - 59.2 \pm 3.1$)$, compared with the corresponding indicators in the KG $(154.3 \pm 9.1$ and $69.3 \pm 2.2)$ and the group of patients after cryosurgical treatment and the use of preventive measures for the prevention of inflammatory and TEC.

Positive reactions of the ethanol test also testify to the normalization of the fibrinolysis system, the number of which decreased in contrast to the indicators before treatment, namely with $97.3 \%$ to $63.6 \%$. Thus, in our opinion, it indicates the lack of negative impact of the applied method of therapy on the hemostasis system and the high efficiency of hysteroscopy combined with endocryosurgical treatment of endo pathology- and myometrium in patients of the perimenopausal period with extragenital pathology.

Ultrasound data obtained three months after treatment indicated the absence of signs of endometrial hyperplasia and a decrease in anterior-posterior dimensions of the uterus.

Cytological findings of aspirates from the uterine cavity, which we got through 3 months after intrauterine cryosurgery, indicated the absence of atypical or precancerous processes.

Based on the results of a comprehensive examination before and after the use of our proposed therapy and prevention of complications in the treatment of combined endo pathology- and myometrium in patients with extragenital pathology, based on the results of dispensary observation of our patients from first to the sixth year, we have been deciding on the criteria for the effectiveness of therapy. 
Thus, by three months and during the following second years of dispensary observation, it should be no complaints, no complaints during the bimanual examination, reducing the size of the uterus (Ultrasound), endometrial thickness 3-4 $\mathrm{mm}$, negative results of the pathomorphological examination of aspirates from the uterine cavity.

So, the effectiveness of the proposed approach to diagnosis, minimally invasive treatment (hysteroscopy and intrauterine cryosurgery), and prevention of TEC and inflammatory complications of endo pathology- and myometrium in patients of the perimenopausal period with extragenital pathology, according to the clinic-laboratory and cytomorphological examination, the results of ultrasound was $82.0 \%$, prevention of TEC and inflammatory complications $100 \%$ cases. According to generally accepted approaches, the effectiveness of treatment was $36.0 \%$, TEC prevention - $96.0 \%$, prevention of inflammatory complications - $78.0 \%$.

\section{REFERENCES}

Sasaki, L. M. P., Andrade, K. R. C., Figueiredo, A. C. M. G., da Silva Wanderley, M., \& Pereira, M. G. (2018). Factors associated with malignancy in hysteroscopically resected endometrial polyps: a systematic review and meta-analysis. Journal of minimally invasive gynecology, 25(5), 777-785.

Vitale, S. G., Haimovich, S., Laganà, A. S., Alonso, L., Sardo, A. D. S., \& Carugno, J. (2021). Endometrial polyps. An evidence-based diagnosis and management guide. European Journal of Obstetrics \& Gynecology and Reproductive Biology.

Sadigov Yu. M. (2019). Hyperplastic Processes an Endometrium at Women of Post-menopausal Age: Clinical-anamnestic Aspects. Family Medicine, (5-6), 153-156. https://doi. org/10.30841/2307-5112.5-6.2019.194893

Engstrom, P. F., \& Meyskens Jr, F. L. (2005). Cancer Prevention Strategies. Principles and Practice of Gynecologic Oncology, 179.

Vovk I.B., \& Ponomareva I.G. (2016) Features of aerobic microflora in women with hyperpro- liferative diseases of the uterus and endometrium. Women's health. №2 (108). p. 102-106.

Korniyenko, S. (2017). Endometrial pathology and reproductive profile of women in late reproductive and premenopausal age. ScienceRise: Medical Science, 37-42.

Fiscella, K., Eisinger, S. H., Meldrum, S., Feng, C., Fisher, S. G., \& Guzick, D. S. (2006). Effect of mifepristone for symptomatic leiomyomata on quality of life and uterine size: a randomized controlled trial. Obstetrics \& Gynecology, 108(6), 1381-1387.

Westin, S. N., Fellman, B., Sun, C. C., Broaddus, R. R., Woodall, M. L., Pal, N., ... \& Yates, M. S. (2021). Prospective phase II trial of levonorgestrel intrauterine device: Nonsurgical approach for complex atypical hyperplasia and early-stage endometrial cancer. American journal of obstetrics and gynecology, 224(2), 191-e1.

Rotenberg, O., Doulaveris, G., Fridman, D., Renz, M., Kaplan, J., Xie, X., \& Goldberg, G. L. (2020). Long-term outcome of postmenopausal women with proliferative endometrium on endometrial sampling. American journal of obstetrics and gynecology, 223(6), 896-e1.

Uglietti, A., Buggio, L., Farella, M., Chiaffarino, F., Dridi, D., Vercellini, P., \& Parazzini, F. (2019). The risk of malignancy in uterine polyps: A systematic review and meta-analysis. European Journal of Obstetrics \& Gynecology and Reproductive Biology, 237, 48-56.

Wise, M. R., Gill, P., Lensen, S., Thompson, J. M., \& Farquhar, C. M. (2016). Body mass index trumps age in decision for endometrial biopsy: cohort study of symptomatic premenopausal women. American journal of obstetrics and gynecology, 215(5), 598-e1.

Shalowitz, D. I., Goodwin, A., \& Schoenbachler, N. (2019). Does surgical treatment of atypical endometrial hyperplasia require referral to a gynecologic oncologist?. American journal of obstetrics and gynecology, 220(5), 460-464.

Korniyenko, S. M. (2015). Infertility and Personality of the late reproductive age patients with the endometrial pathology. Women's health, (4), 126-128. 
Pham, T. T., Chen, Y. B., Adams, W., Wolff, B., Shannon, M. B., \& Mueller, E. R. (2019). 21: Characterizing anxiety at the first encounter in women presenting to the urogynecology clinic: the Café Study. American Journal of Obstetrics \& Gynecology, 220(3), S719-S720.

Hervé, F., Katty, A., Isabelle, Q., \& Céline, S. (2018). Impact of uterine fibroids on quality of life: a national cross-sectional survey. European Journal of Obstetrics \& Gynecology and Reproductive Biology, 229, 32-37.

Benyuk, V. O., Kravchenko, Y. V., Kovaliuk, T. V., Beniuk, S. V., \& Usevych, I. A. (2020). Modern treatment of endometrial local hyperplastic processes from positions of the pathogenic approach. Reproductive Endocrinology, (52), 28-32.

Horak, P., Mara, M., Dundr, P., Kubinova, K., Kuzel, D., Hudecek, R., \& Chmel, R. (2012). Effect of a selective progesterone receptor modulator on induction of apoptosis in uterine fibroids in vivo. International journal of endocrinology, 2012.

Clarke, M. A., Long, B. J., Sherman, M. E., Lemens, M. A., Podratz, K. C., Hopkins, M. R., ... \& Wentzensen, N. (2020). Risk assessment of endometrial cancer and endometrial intraepithelial neoplasia in women with abnormal bleeding and implications for clinical management algorithms. American journal of obstetrics and gynecology, 223(4), 549-e1.

Gladchuk, I. Z., Rozhkovskaya, N. M., \& Kosey, T. V. (2016). Modern surgical technologies in the treatment of uterine fibroids (literature review and own data). Collection of scientific works of the Association of Obstetricians and Gynecologists of Ukraine, 2(38), 123-9.

Golyanovskyy, O. V., \& Budchenko, M. A. (2018). Prevention of pregnancy and sickness after a sustained method of conservative myomectomy. Reports of Vinnytsia National Medical University, 22(1), 152-155.

Giannella, L., \& Paganelli, S. (2017). Abnormal uterine bleeding in premenopausal women and the role of body mass index. American Journal of Obstetrics \& Gynecology, 216(5), 533.

Harvey, L., Slocum, P., Heft, J., Mokshagundam, S., Crispens, M., \& Zimmerman, C. W.
(2018). 71: Route of hysterectomy for endometrial pathology in superobese patients. American Journal of Obstetrics \& Gynecology, 218(2), S936-S937.

Tatarchuk T.F., Kosey N.V., Zanko O.V., Retunska I.M. (2018) Prognostication occurrence hyperproliferative diseases endometrium in women in postmenopausal. Collection of scientific works of the Association of Obstetricians-gynecologists of Ukraine..№2 (42). p. 179-183.

Maggi, R., Cariboni, A. M., Marelli, M. M., Moretti, R. M., Andre, V., Marzagalli, M., \& Limonta, P. (2016). GnRH and GnRH receptors in the pathophysiology of the human female reproductive system. Human reproduction update, 22(3), 358-381.

Tatarchuk T.F., Kosey N.V., Zanko O.V., Retunska I.M. (2018) Prediction of the occurrence of hyperproliferative diseases of the endometrium in postmenopausal women. Collection of scientific works of the Association of Obstetricians-gynecologists of Ukraine.№2 (42). p. $179-183$.

Beelen, P., van den Brink, M. J., Herman, M. C., Geomini, P. M., Dekker, J. H., Duijnhoven, R. G., ... \& Bongers, M. Y. (2021). Levonorgestrel-releasing intrauterine system versus endometrial ablation for heavy menstrual bleeding. American Journal of Obstetrics and Gynecology, 224(2), 187-e1.

Di Spiezio Sardo, A., Di Carlo, C., Minozzi, S., Spinelli, M., Pistotti, V., Alviggi, C., ... \& Bifulco, G. (2016). Efficacy of hysteroscopy in improving reproductive outcomes of infertile couples: a systematic review and meta-analysis. Human reproduction update, 22(4), 479-496.

Senchuk A.Y., Zakordonets R.M. (2017) The state of the hemostasis system in patients with precancerous diseases of the endometrium.Collection of scientific works of the Association of Obstetricians-gynecologists of Ukraine. №2 (40). p. 236-240.

\section{Corresponding author:}

Anatolii Ya. Senchuk, 0509453723@ukr.net

Manuscript was recieved on 17 September; accepted for publication on 21 October. 